\title{
ON AN EXTREMAL PROPERTY OF DOOB'S CLASS
}

BY

\author{
J. S. HWANG ${ }^{1}$
}

\begin{abstract}
Recently, we have solved a long open problem of Doob (1935). To introduce the result proved here, we say that a function $f(z)$ belongs to Doob's class $D$, if $f(z)$ is analytic in the unit disk $U$ and has radial limit zero at an endpoint of some arc $R$ on the unit circle such that $\lim _{\inf } f_{n \rightarrow \infty}\left|f\left(P_{n}\right)\right|$ $>1$, where $\left\{P_{n}\right\}$ is an arbitrary sequence of points in $U$ tending to an arbitrary interior point of $R$.

With this definition, our main result is the following extremal property of Doob's class.

Throrem. $\inf _{f \in D}\|f\|=2 / e$, where $\|f\|=\sup _{z \in U}\left(1-|z|^{2}\right)\left|f^{\prime}(z)\right|$.
\end{abstract}

1. Introduction. Let $f(z)$ be a function analytic in the unit disk $U=\{z$ : $|z|<1\}$. Following Doob [2], we shall say that a function $f \in K(\rho)$, if it satisfies

$$
f(0)=0,
$$

and for some $\operatorname{arc} R$ on the unit circle $\partial U$ with measure $|R| \geqslant \rho>0$,

$$
\liminf _{n \rightarrow \infty}\left|f\left(P_{n}\right)\right| \geqslant 1,
$$

where $\left\{P_{n}\right\}$ is an arbitrary sequence of points in $U$ converging to an arbitrary interior point of $R$.

Instead of the normalization (1), we have introduced in [4], the notion of Doob's class $D(\rho)$, namely, a function $f \in D(\rho)$, if it satisfies (2) and the following:

$$
f(z) \text { has radial limit } 0 \text { at an endpoint of } R \text {. }
$$

The number $\rho$ is only important for considering the covering problem, but not for the extremal problem. Therefore, in this paper, we shall denote Doob's class $D(\rho)$ by $D$.

As was introduced by Pommerenke (see [1]) a function $f(z)$ is called a Bloch function, if the following Bloch norm is finite:

$$
B_{f}=\|f\|=\sup _{z \in U}\left(1-|z|^{2}\right)\left|f^{\prime}(z)\right| \text {. }
$$

Received by the editors April 8, 1978 and, in revised form, August 4, 1978.

AMS (MOS) subject classifications (1970). Primary 30A42; Secondary 30A72, 30A74.

Key words and phrases. Extremal property and Doob's class.

'I am indebted to Professor Timoney for his many valuable comments and corrections, and in particular for his disproving my conjectures in my manuscript. 
This quantity was studied much earlier in 1942 by Seidel and Walsh [8]. For this reason, we shall call

$$
\alpha(A)=\inf _{f \in A}\|f\|,
$$

where $A$ is a class of functions, the Seidel and Walsh constant for the class $(A)$. With this definition, our main result is the following one about the Seidel and Walsh constant for Doob's class.

THEOREM 1. $\alpha(D)=2 / e$.

2. Minimum principle. Instead of normal functions developed by Lehto and Virtanen [5, Theorem 6], we shall now establish the following minimum principle.

LEMMA 1. Let $f(z)$ be a Bloch function in $U$ and let $G$ be a subdomain of $U$ bounded by two analytic curves $\nu$ and $\nu^{\prime}$ such that

$$
\begin{aligned}
|f(z)| & \geqslant x, & & \text { for } z \in \nu, \\
& \geqslant y, & & \text { for } z \in G, \\
|f(P)| & =y, & & \text { for some interior point } P \in \nu^{\prime} .
\end{aligned}
$$

Let $\omega(z)$ be the harmonic measure on $G$ which is equal to 1 on $\nu$ and vanishes on $\nu^{\prime}$. Then we have

$$
1 / x>(1 / y) e^{-B} f^{\lambda} P^{/ y}, \quad \lambda_{P}=1 /\left(\left(1-|P|^{2}\right) \partial \omega(P) / \partial n\right),
$$

where $\partial \omega / \partial n$ is the normal derivative with respect to the inner normal of $\nu^{\prime}$.

Proof. Let $g(z)=1 / f(z)$, then by (6), we have

$$
\begin{aligned}
|g(z)| & \leqslant 1 / x, & & \text { for } z \in \nu, \\
& \leqslant 1 / y, & & \text { for } z \in G, \\
|g(P)| & =1 / y, & & \text { for some } P \in \nu^{\prime} .
\end{aligned}
$$

It follows from (4) that for $z \in G$,

$$
\frac{1}{y}-|g(z)|<\left|\int_{z}^{P} g^{\prime}(w) d w\right|<B_{f} \int_{z}^{P} \frac{|d w|}{|f(w)|^{2}\left(1-|w|^{2}\right)}=B(z) .
$$

Let $h(z)=(1 / y)(y / x)^{\omega(z)}$, for $z \in G$; then clearly we have $|g(z)|<h(z)$, for $z \in \nu \cup \nu^{\prime}$. Owing to the ordinary maximum principle, we find that

$$
|g(z)| \leqslant h(z), \text { for all } z \in G .
$$

Combining (9) and (10), we get for $z \in G$,

$$
\log (1 / y-B(z)) \leqslant \log |g(z)| \leqslant \log (1 / y)+\omega(z) \log (y / x) .
$$


By taking the normal derivative, we obtain

$$
-\frac{\partial B(P)}{\partial n} /\left(\frac{1}{y}-B(P)\right) \leqslant \frac{\partial \omega(P)}{\partial n} \log \frac{y}{x} .
$$

Since $B(P)=0$ and $|f(P)|=y$, it follows that $\partial B(P) / \partial n=B_{f} /\left(y^{2}(1-\right.$ $\left.\left.|P|^{2}\right)\right)$. By substituting into (11), we conclude the desired result (7).

If we denote $m=1 / x$ and $M=1 / y$, then the above result is similar to [5, Theorem 6] in which the number $1 / M$ in the second term of the exponent disappears, see [5, (7), p. 58]. By the same consideration as [5, (12), p. 60], we obtain the following result similar to [4, Lemma 13].

LEMMA 2. Let $\beta$ be the interior angle of a moon shape domain $G$ bounded by an arc on $\partial U$ and its subtended chord. If $f(z)$ is a Bloch function in $U$ such that (6) holds, then we have

$$
1 / x \geqslant(1 / y) e^{-B_{f} \beta / 2 y \sin \beta} .
$$

3. Proof of Theorem 1. We first want to prove the upper bound of Seidel and Walsh constant of Doob's class. To do this, it is sufficient to find a function $f \in D$ with $\|f\|=2 / e$. By the same argument as [4, Lemma 8], we can see that the function $f_{1}(z)=e^{-(1+z) /(1-z)} \in D$ and $\left\|f_{1}\right\|=2 / e$. This establishes that

$$
\alpha(D) \leqslant 2 / e .
$$

Conversely, we shall show that

$$
\|f\| \geqslant 2 / e, \text { for any } f \in D .
$$

Let $f(z)$ be an arbitrary function in $D$. If $\|f\|=\infty$, then (14) is true and therefore we may assume that $\|f\|<\infty$. It follows that the function $f(z)$ is normal in $U$. By virtue of [5, Theorem 5], we know that the notions of radial limits and angular limits are equivalent for normal functions. Let $g(z)=$ $1 / f(z)$, then by (3), we find that $g(z)$ has angular limit $\infty$ at an endpoint of the arc $R$, say, at $z=1$.

In view of [4, Lemma 12], we can see that for any $1<M<\infty$, there is a moon shape domain $G$ arbitrarily small and arbitrarily close to $z=1$ such that

$$
\max |g(z)|=M \quad \text { or } \min |f(z)|=1 / M, \text { for } z \in G \text {. }
$$

We shall now apply Lemma 2 . By substituting $x=1$ and $y=1 / M$ into (12), we obtain

$$
1 \geqslant M e^{-B} f^{M \beta / 2 \sin \beta}=M e^{-B M \lambda},
$$

where $B=B_{f}$ and $\lambda=\lambda(\beta)=\beta / 2 \sin \beta$.

Clearly, the right-hand side of (16) attains its largest value $1 / e B \lambda$, at $M^{*}=1 / B \lambda$. 
Since $G$ can be taken arbitrarily small, hence by letting $\beta \rightarrow 0$, we get $\lambda=\lim _{\beta \rightarrow 0} \lambda(\beta)=1 / 2$. By (15), we can choose $M^{*}=2 / B$ and then substitute into (16), we obtain $1 \geqslant 2 / e B$ or $B_{f} \geqslant 2 / e$. This concludes (14) and $\alpha(D) \geqslant 2 / e$. Combining with (13) yields the result and the proof is complete.

4. Seidel and Walsh constant for $A_{i}$-class. In this section, we want to summarize all results similar to those in [4]. The proof is just the same and we omit it. Following Seidel [7], we denote $A=\left\{f:\left|f\left(e^{i \theta}\right)\right|=1\right.$, a.e. on $\left.\partial U\right\}$. As was classified in [4], we denote those subclasses of $A$ by $A_{0}, A_{\infty}, A_{1}, A_{I}$, and $A(\rho)$ to be the finite Blaschke products, infinite Blaschke products, singular inner functions, isolated singular functions, and $A(\rho)=A \cap K(\rho)$, respectively. By the same argument as [4, Lemmas 5-10, and 14], we can easily obtain the following Seidel and Walsh constant for $A_{i}$-class.

TheOREM 2. $\alpha\left(A_{i}\right)=2 / e$, for $i=0, \infty, 1, I$, and $\rho$.

Notice that $A_{0}$ and $A_{\infty}$ are not subclasses of $D$, so that Theorem 2 is not a corollary of Theorem 1.

5. The O. K. constant of Doob's class. Instead of the Bloch norm, we shall now consider the following $\mathrm{O}$. K. norm, see [4],

$$
\|f\|_{\text {O.K. }}=\sup _{z \in U}\left(1-|z|^{2}\right)\left|f^{\prime}(z)\right| /\left(1+|f(z)|^{2}\right) \text {. }
$$

In [4], we prove that

$$
C(A(\rho))=\inf _{f \in A(\rho)}\|f\|_{\mathrm{o} . \mathrm{K} .}=C_{1}, \text { for } 0<\rho \leqslant 2 \pi,
$$

where $C_{1}$ is a number between $1 / 2$ and $2 / e$ which is the unique solution of

$$
F(C)=\left[1+\left(1+C^{2}\right)^{1 / 2}\right] / C e^{\left(1+C^{2}\right)^{1 / 2}}=1
$$

This result was proved only for the Seidel class $(A)$. By the same method as Theorem 1, we can now easily obtain the following generalization.

THEOREM 3. $C(D)=\inf _{f \in D}\|f\|_{\text {O.K. }}=C_{1}$.

6. Doob's problem. In [3, Theorems 1 and 2], we have proved the following results which solve two problems of Doob [2, p. 120].

THEOREM 4. If $f \in K(\rho)$, then $\|f\|>1 / N(\rho)$, where $N(\rho)=\max \left(\pi e^{\sqrt{2}} / 2\right.$, $e \log 1 /(1-\cos \rho))$.

THEOREM 5. If $f \in K(\rho)$, then the range of $f(z)$ covers the interior of some circle of radius $k(\rho)=1 /(16 e \log 1 /(1-\cos \rho))$.

Instead of using Lehto and Virtanen's theorem [1, p. 30], we can also prove these two theorems from Lemma 2. The method is the same as that in [3] and 
we omit the details, but point out that, in equation (11) of [3],

$$
h(\lambda)=(1+\varepsilon) \exp [(\beta / n \sin \beta)(\lambda+1 / \lambda)]-\lambda ;
$$

we can now omit the term $1 / \lambda$ in the exponent. Therefore, with a slight simplification, we can obtain the same assertion.

7. The range of Doob's class. By the same method as Theorem 5 , see [3, Theorem 2], we can also obtain the following covering property.

THEOREM 6. If $f \in D$, then the range of $f(z)$ covers the interior of some circle of radius $1 / 8 e$.

Naturally, we may ask whether this theorem is still true if condition (3) is omitted. The answer turns out to be no, as will be seen from the following function, see [3, Example 1]: $f_{n}(z)=\left(z^{n}+1\right) / n^{2}+1$. This function satisfies condition (2) at every point on $\partial U$. However, the range of $f_{n}(z)$ does not cover any circle of positive radius. Of course, $f_{n}$ can also show the necessity of condition (3) in all previous theorems.

8. The range of Seidel's class. The constant $1 / 8 e$ in Theorem 6 can be improved to be $1 / 2$ if we restrict to the Seidel's subclass $\left(A_{I}\right)$ which is also a subclass of Doob. Of course, there are two kinds of subclasses of Seidel's class which are not included in the Doob's class, namely, Blaschke products and singular inner functions whose singularities are dense on the circle. In contrast to Seidel [6, Theorem 4], we shall now prove the following covering property.

THEOREM 7. If $f \in A_{I}$, then the range of $f(z)$ covers the interior of some circle of radius $1 / 2$.

Proof. We may assume that the isolated singularity of $f(z)$ is located at $z=1$. We shall prove that the range of $f$ covers $U$ except at most one point. To do this, there are three cases to be considered, either $f$ is a Blaschke product whose zeros tend to $z=1$ or $f$ is a singular inner function or both.

In the first case, the result is due to the aforementioned theorem of Seidel. The last two cases can also be proved by the same argument as that used by Seidel, namely, by considering the functional equation $f(1 / \bar{z})=1 / \overline{f(z)}$, where $\left|f\left(e^{i \theta}\right)\right|=1,0<|\theta|<\delta$, we find that the function $f$ can be continued analytically across the arc $R$ and therefore by Picard's theorem, we conclude that the range of $f$ covers $U$ except at most one point, say, $P$. It follows that this range $U-P$ covers the interior of some circle of radius at least $1 / 2$. This completes the proof.

Notice that the above theorem is best possible in the sense that this constant $1 / 2$ cannot be improved. To see this, let us recall the function $f_{1}(z)$ defined in the proof of Theorem 1. From there, we can see that the range of $f_{1}$ 
covers $U$ and saves the point at $z=0$, so that the range $U-0$ covers the interior of some circle of radius exactly $1 / 2$.

\section{REFERENCES}

1. J. M. Anderson, J. Clunie and Ch. Pommerenke, On Bloch functions and normal functions, J. Reine Angew. Math. 270 (1974), 12-37.

2. J. L. Doob, The ranges of analytic functions, Ann. of Math. 36 (1935), 117-126.

3. J. S. Hwang, On two problems of Doob about the ranges of analytic functions (to appear).

4. $\longrightarrow$, On the O.K. constant of Doob and Seidel's class. (to appear).

5. O. Lehto and K. J. Virtanen, Boundary behaviour and normal meromorphic functions, Acta Math. 97 (1957), 47-65.

6. W. Seidel, On the cluster values of analytic functions, Trans. Amer. Math. Soc. 34 (1932), $1-21$.

7. 36 (1934), 201-226.

8. W. Seidel and J. L. Walsh, On the derivatives of functions analytic in the unit circle and their radii of univalence and p-valence, Trans. Amer. Math. Soc. 52 (1942), 128-216.

Department of Mathematics, McMaster Universtry, Hammton, ONTARio L8S 4K1, Canada

Current address: Institute of Mathematics, Academia Sinica, Taipei, Taiwan 